\title{
Secagem por atomização de polpa de amora-preta usando maltodextrina como agente carreador
}

\author{
Spray drying of blackberry pulp using maltodextrin as carrier agent
}

\section{Autores | Authors}

\ Cristhiane Caroline FERRARI

Instituto de Tecnologia de Alimentos (ITAL) Centro de Tecnologia de Frutas e Hortaliças (FRUTHOTEC)

Av. Brasil, 2880

CEP: $13.070-178$

Campinas/SP - Brasil email: cferrari@ital.sp.gov.br

Caio Pereira RIBEIRO José Maurício de AGUIRRE

Instituto de Tecnologia de Alimentos Centro de Tecnologia de Frutas e Hortaliças Campinas-SP, Brasil e-mail: caiopr@gmail.com maguirre@ital.sp.gov.br

$\triangle$ Autor Correspondente / Corresponding Author

Recebido / Received: 28/03/2011 Aprovado / Approved: 25/01/2012

Publicado / Published: jun./2012

\section{Resumo}

O objetivo deste trabalho foi avaliar a influência da temperatura do ar de secagem (160 ou $180{ }^{\circ} \mathrm{C}$ ) e da concentração de maltodextrina (5, 15 ou $25 \%$ ) sobre as características físico-químicas do suco de amora-preta em pó, produzido por spray drying. O produto final foi analisado quanto ao teor de umidade e de antocianinas, higroscopicidade, atividade de água, cor, distribuição do tamanho das partículas e microestrutura. Temperaturas de secagem mais altas resultaram na redução da umidade, da atividade de água e do conteúdo de antocianinas dos pós, além da formação de partículas maiores e mais higroscópicas. A temperatura teve um efeito significativo na diminuição dos valores dos parâmetros de cor $L^{*}$ e $\mathrm{C}^{*}$ somente nos ensaios realizados com $25 \%$ de maltodextrina. Em relação à microestrutura, as partículas produzidas à temperatura de $180^{\circ} \mathrm{C}$ apresentaram superfície lisa e uma maior uniformidade em relação às amostras secas a $160^{\circ} \mathrm{C}$. O aumento da concentração de maltodextrina resultou na formação de partículas com menor umidade e higroscopicidade. Além disso, esses pós apresentaram menor teor de antocianinas, em função da diluição dos pigmentos da fruta, o que levou ao aumento da luminosidade e à redução do croma e do ângulo de tom das amostras. O ensaio realizado à temperatura de $160^{\circ} \mathrm{C}$ com $5 \%$ de maltodextrina foi o mais efetivo na manutenção do teor de antocianinas dos pós, visto que a retenção desses pigmentos foi em torno de $80 \%$.

Palavras-chave: Amora-preta em pó; Tamanho de partículas; Antocianinas; Higroscopicidade; Microestrutura.

\section{Summary}

The aim of this work was to evaluate the influence of inlet air temperature $\left(160\right.$ or $180{ }^{\circ} \mathrm{C}$ ) and maltodextrin concentration (5, 15 or $25 \%$ ) on the physicochemical characteristics of powdered blackberry juice produced by spray drying. The final product was analysed with respect to moisture content, hygroscopicity, water activity, anthocyanin content, colour, particle size distribution and microstructure. Higher inlet air temperatures resulted in decreases in moisture content, water activity and anthocyanin retention, besides the formation of larger sized, more hygroscopic particles. Temperature only showed a significant effect on the reduction of the $L^{*}$ and $C^{*}$ values for treatments carried out with $25 \%$ maltodextrin concentration. Regarding the microstructure, particles produced at $180^{\circ} \mathrm{C}$ showed smooth surfaces and a greater degree of uniformity in comparison with samples spray dried at $160{ }^{\circ} \mathrm{C}$. An increase in maltodextrin concentration promoted the production of less hygroscopic particles with lower moisture content. Furthermore, these powders showed lower anthocyanin contents, due to dilution of the fruit pigments, leading to an increase in the $L^{*}$ values and reduction in the chroma and hue angle values. The treatment carried out at $160{ }^{\circ} \mathrm{C}$ with $5 \%$ maltodextrin was the most effective in maintaining the anthocyanin content of the powder, with a pigment retention of about $80 \%$.

Key words: Blackberry powder; Particle size; Anthocyanin; Hygroscopicity; Microstructure. 


\section{Introdução}

A amora-preta, fruta originária da Ásia, atualmente é produzida em países da Europa, da América do Norte e da América do Sul, crescendo em regiões de clima frio. Além das suas características atrativas de cor e sabor, várias pesquisas recentes têm destacado as propriedades benéficas da amora-preta (antiinflamatória e anticarcinogênica) e a sua função antioxidante (VIZZOTTO, 2008). Essas propriedades funcionais estão relacionadas à alta concentração de antocianinas presentes na fruta (FERREIRA, 2008; ACOSTA-MONTOYA et al., 2010). Entretanto, a amorapreta apresenta uma vida pós-colheita curta, em função da sua elevada taxa respiratória e da estrutura frágil, sendo necessária a utilização de técnicas de processamento adequadas para prolongar o período de conservação das frutas, preservando a sua qualidade.

A utilização da secagem por atomização (spray drying) da polpa de amora-preta representa uma alternativa viável para a sua conservação. Além disso, como a comercialização da fruta está restrita à sua época de safra, o processo de spray drying permite o aumento da vida útil do produto e uma maior estabilidade, podendo o consumidor ter acesso a esse produto durante todo o ano. A secagem por atomização é um processo contínuo, que se caracteriza pela transformação de um líquido em um produto seco, na forma de pó, por meio de um tempo de secagem relativamente curto. Esse líquido é atomizado, utilizando-se um sistema centrífugo ou de alta pressão, pelo qual as gotículas atomizadas entram em contato com um fluxo de ar quente. Assim, há uma rápida evaporação, que permite manter baixa a temperatura do produto final, possibilitando a secagem de produtos sensíveis ao calor sem afetar excessivamente sua qualidade (RÉ, 1998).

No caso dos sucos de frutas, o alto conteúdo de açúcares pode acarretar a obtenção de produtos com alta pegajosidade e higroscopicidade, diminuindo o rendimento do processo. Portanto, é fundamental a utilização de agentes carreadores com alto peso molecular - como polímeros e gomas - antes da atomização, visando facilitar o processo de secagem e as operações de transporte e armazenamento. A maltodextrina é o agente carreador comumente empregado na secagem por atomização, em função de sua baixa higroscopicidade, alta solubilidade em água fria e baixo custo (BHANDARI et al., 1997).

Neste contexto, o objetivo deste trabalho foi estudar a influência da temperatura do ar de secagem e da concentração de maltodextrina sobre as características físico-químicas do suco de amorapreta em pó produzido por spray drying, avaliando-se a umidade, a higroscopicidade, a atividade de água, a cor, a retenção de antocianinas, além do tamanho e da microestrutura das partículas obtidas no processo.

\section{Material e métodos}

\subsection{Material}

\subsubsection{Matéria-prima}

Foi utilizada polpa de amora-preta adquirida da empresa De Marchi Indústria e Comércio de Frutas Ltda (Jundiaí-SP, Brasil). A polpa foi armazenada em um freezer, modelo DF46, marca Electrolux (São Paulo-SP, Brasil), à temperatura de $-18^{\circ} \mathrm{C}$, sendo descongelada de acordo com a necessidade para cada ensaio.

\subsubsection{Agente carreador}

Utilizou-se a maltodextrina Maltogill@ 20DE (Cargill, Uberlândia-MG, Brasil).

\subsection{Métodos}

\subsubsection{Preparo da amostra}

Inicialmente, a polpa de amora-preta foi descongelada em geladeira $\left(4-5{ }^{\circ} \mathrm{C}\right)$ por 18 horas. Em seguida, o agente carreador foi adicionado à polpa nas concentrações pré-determinadas de 5, 15 ou 25\% (p/p). A mistura foi homogeneizada em moinho coloidal modelo REX (marca Meteor, São Paulo-SP, Brasil) à temperatura ambiente $\left(25^{\circ} \mathrm{C}\right)$ até a sua completa dissolução.

\subsubsection{Caracterização físico-química da matéria-prima}

A matéria-prima foi caracterizada quanto à composição química centesimal, sendo determinados os teores de umidade, cinzas, proteínas, lipídeos, açúcares (redutores e totais) e acidez total titulável, de acordo com as metodologias da A.O.A.C. (HORWITZ, 2006). As características físicas avaliadas foram sólidos solúveis (medidos em refratômetro de bancada, modelo 10450, marca American Optical Abbe, Buffalo, E.U.A.) e pH (medido em potenciômetro, modelo DM-20, marca Digimed, São Paulo-SP, Brasil). O teor de antocianinas da matéria-prima também foi determinado, de acordo com a metodologia descrita no item 2.2.4, além do conteúdo de compostos fenólicos totais, por meio do método espectrofotométrico de Folin Ciocalteau, descrito por Kiralp e Toppare (2006). Todas as análises foram realizadas em triplicata.

\subsubsection{Ensaios de secagem}

A secagem por atomização foi realizada em mini spray dryer - modelo B-290, marca Buchi (Flawil, Suíça), com capacidade evaporativa de 1 L.h ${ }^{-1}$ de água. O processo foi conduzido em fluxo co-corrente, utilizando-se um atomizador duplofluido de 0,7 mm de diâmetro, fluxo de ar de secagem de $35 \mathrm{~m}^{3} \cdot \mathrm{h}^{-1}$ (CNTP) - o que 
equivale a $100 \%$ da capacidade máxima do aspirador -, fluxo de ar comprimido de 473 L.h $h^{-1}$ e pressão relativa de atomização de 5 bar. A alimentação do secador foi realizada à temperatura ambiente, através de uma bomba peristáltica, utilizando-se $30 \%$ da sua velocidade máxima, o que correspondeu a uma vazão mássica média de $7 \mathrm{~g} \cdot \mathrm{min}^{-1}$. A temperatura de saída do ar foi também monitorada. As temperaturas do ar de secagem utilizadas foram 160 e $180^{\circ} \mathrm{C}$, enquanto que as concentrações de maltodextrina empregadas foram 5, 15 e 25\% (p/p), totalizando seis ensaios. Após o processo, as amostras foram armazenadas em potes herméticos dentro de dessecadores, até o momento da realização das análises descritas no item 2.2.4.

\subsubsection{Análises dos produtos em pó}

\subsubsection{Umidade}

Determinada em estufa a vácuo a $70{ }^{\circ} \mathrm{C}$, por 24 horas (HORWITZ, 2006).

\subsubsection{Higroscopicidade}

Foi avaliada de acordo com a metodologia proposta por Cai e Corke (2000), com algumas modificações. Colocou-se cerca de $1 \mathrm{~g}$ de cada amostra em um recipiente hermético contendo uma solução saturada de $\mathrm{NaCl}$ (umidade relativa de $75,29 \%$ ) a $25{ }^{\circ} \mathrm{C}$ e, após uma semana, as amostras foram pesadas, sendo a higroscopicidade expressa como g de umidade adsorvida por $100 \mathrm{~g}$ de massa seca da amostra $\left(\mathrm{g} .100 \mathrm{~g}^{-1}\right)$.

\subsubsection{Atividade de água}

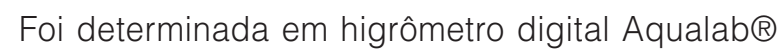
3TE (Decagon Devices Inc, Pullman, EUA), a $25^{\circ} \mathrm{C}$.

\subsubsection{Antocianinas}

O teor de antocianinas no pó e na matéria-prima foi determinado por meio da metodologia do $\mathrm{pH}$ diferencial (HORWITZ, 2006), sendo que a extração foi feita com solução de acetona (70\%). Os valores de absorbância foram medidos em espectrofotômetro modelo 700Plus (Femto, São Paulo-SP, Brasil) nos comprimentos de onda de 520 e $700 \mathrm{~nm}$. O teor de antocianinas foi calculado usando-se o coeficiente de extinção molar de $26900 \mathrm{~L}$. $\mathrm{cm}^{-1}$. mol referente à cianidina-3-glicosídeo, antocianina majoritária presente na amora-preta. Para a avaliação da retenção de antocianinas, determinou-se também a quantidade desses pigmentos na mistura alimentada no spray dryer.

\subsubsection{Cor}

A cor foi avaliada por meio do sistema de leitura de três parâmetros, o CIELAB, proposto pela Comission
Internacionale de l'Eclairage (CIE), em 1971. Os parâmetros $L^{*}, a^{*} e b^{*}$ foram fornecidos pelo colorímetro modelo CR400 (Konica Minolta, Osaka, Japão), no qual $L^{*}$ define a luminosidade $\left(L^{*}=0\right.$ preto e $L^{*}=100$ branco) e $a^{*} e b^{*}$ são responsáveis pela cromaticidade $\left(+a^{*}\right.$ vermelho $e-a^{*}$ verde, $+b^{*}$ amarelo $e-b^{*}$ azul). Com esses parâmetros, foram avaliadas as coordenadas cilíndricas $\mathrm{C}^{*}$ e $\mathrm{H}^{*}$, sendo que $\mathrm{C}^{*}$ define o croma e $\mathrm{H}^{\star}$, o ângulo de tom, a partir das Equações 1 e 2 (CALVO e DURÁN, 1997).

$$
\begin{aligned}
& C^{*}=\left(a^{* 2}+b^{* 2}\right)^{1 / 2} \\
& H^{*}=\arctan \left(\frac{b^{*}}{a^{*}}\right)
\end{aligned}
$$

\subsubsection{Distribuição do tamanho de partículas}

A distribuição do tamanho das partículas foi determinada em um analisador de tamanho de partículas por difração a laser (Laser Scattering Spectrometer Mastersizer S, modelo MAM 5005 - Malvern Instruments Ltd., Malvern, Inglaterra), utilizando-se isopropanol como líquido sedimentador, uma vez que a solubilização das partículas não ocorre nesse líquido. Um ultrassom acoplado ao equipamento foi utilizado para aumentar a dispersibilidade da amostra. O diâmetro médio foi determinado com base no diâmetro médio de uma esfera de mesmo volume (diâmetro de Brouckere, D[4,3]), geralmente utilizado para caracterizar partículas de pó.

\subsubsection{Microestrutura}

O estudo da morfologia das partículas foi realizado por meio da microscopia eletrônica de varredura (MEV), de acordo Tonon et al. (2008). As amostras foram fixadas em porta-espécimes metálicos, com uma fita adesiva de dupla face condutora convencional. Em seguida, foram metalizadas com uma liga de ouro/paládio, em um metalizador Sputter Coater POLARON, modelo SC7620 (VG Microtech, Ringmer, Inglaterra), a uma taxa de recobrimento de $0,51 \AA . \mathrm{s}^{-1}$, por 180 segundos, com corrente de 3-5 mA, 1 Volt e 8-9.10-2 mbar. As amostras foram então observadas em um microscópio eletrônico de varredura, modelo LEO440i (LEO Electron Microscopy, Oxford, Inglaterra), operando com tensão de aceleração de $20 \mathrm{kV}$ e corrente do feixe de elétrons de 150pA.

\subsubsection{Análise estatística}

Todas as análises foram feitas em triplicata, com exceção das determinações da distribuição do tamanho de partículas e dos parâmetros de cor, em que foram realizadas nove leituras de cada amostra. Os resultados foram avaliados estatisticamente por meio da Análise de Variância (ANOVA) e do Teste de Tukey no nível de 5\% de significância, com o auxílio do programa Statistica ${ }^{\circledR}$ 8.0 (StatSoft Inc., Tulsa, EUA). 


\section{Resultados e discussão}

\subsection{Caracterização físico-química da matéria-prima}

A composição físico-química da polpa de amorapreta utilizada na secagem em spray dryer é apresentada na Tabela 1.

Os resultados mostram que a polpa de amora-preta é constituída principalmente de água e açúcares, tendo uma quantidade muito pequena de lipídios e cinzas, não tendo sido detectada quantidade significativa de proteínas pelo método de análise empregado. Além disso, a polpa de amora-preta apresentou um alto teor de antocianinas e compostos fenólicos. Ferreira (2008) encontrou um teor de antocianinas totais para a amorapreta de 90,5 mg. $100 \mathrm{~g}^{-1}$ amostra e um teor de compostos fenólicos totais de 241,7 mg. $100 \mathrm{~g}^{-1}$ (expresso em ácido gálico), superiores aos obtidos no presente trabalho, o que pode ser atribuído ao fato de que esta autora utilizou a fruta fresca em seu estudo.

Tabela 1. Composição físico-química da polpa de amora-preta.

\begin{tabular}{lr}
\multicolumn{1}{c}{ Componente } & \multicolumn{1}{c}{ Conteúdo } \\
\hline Umidade (\% b.u.) & $91,96 \pm 0,14$ \\
Açúcares redutores & $5,35 \pm 0,26$ \\
(g de açúcar redutor. $100 \mathrm{~g}^{-1}$ b.u.) & \\
Açúcares totais & $6,49 \pm 0,15$ \\
(g de açúcar invertido.100 $\mathrm{g}^{-1}$ b.u.) & \\
Lipídios (g. $100 \mathrm{~g}^{-1}$ b.u.) & $0,10 \pm 0,00$ \\
Cinzas (g.100 g $\mathrm{g}^{-1}$ b.u.) & $0,20 \pm 0,00$ \\
Teor de sólidos solúveis ( ${ }^{\circ}$ Brix) & $6,77 \pm 0,12$ \\
pH & $3,31 \pm 0,02$ \\
Acidez (\% ác. cítrico. $100 \mathrm{~g}^{-1}$ b.u.) & $0,76 \pm 0,02$ \\
Antocianinas (mg. $100 \mathrm{~g}^{-1}$ b.u.) & $77,90 \pm 2,10$ \\
Compostos fenólicos totais & $210,71 \pm 4,24$ \\
(mg ácido gálico. $100 \mathrm{~g}^{-1}$ b.u.) & \\
\hline
\end{tabular}

\subsection{Análises dos produtos em pó}

A Tabela 2 mostra os resultados da análise de variância (ANOVA). Na medida em que $p<0,05$ para todas as características físico-químicas dos pós avaliadas, os tratamentos apresentam diferença significativa entre si, sendo os resultados dos testes de média de Tukey exibidos nas Tabelas 3-5.

\subsubsection{Teor de umidade, higroscopicidade e atividade de água}

Na Tabela 3, são apresentados os resultados relativos à umidade, à higroscopicidade e à atividade de água das amostras, bem como os valores da temperatura do ar de saída.

Ao se observar a Tabela 3, é possível afirmar que a temperatura de secagem e a concentração de maltodextrina tiveram efeito significativo $(p \leq 0,05)$ na umidade e na higroscopicidade. No caso da atividade de água, apenas a temperatura de secagem influenciou significativamente nos resultados, havendo uma redução da atividade de água com o aumento da temperatura (Tabela 3). Além disso, todas as amostras apresentaram valores de atividade de água inferiores a 0,3, indicando estabilidade microbiológica dos pós obtidos por spray drying. O aumento da temperatura do ar de secagem e da concentração de maltodextrina resultou na diminuição da umidade das amostras. Quek et al. (2007) e Moreira et al. (2009) também obtiveram amostras menos úmidas com o aumento da temperatura em seus trabalhos com suco de melancia e suco de acerola em pó, respectivamente, produzidos por spray drying, utilizando-se maltodextrina como agente carreador. O uso de temperaturas mais altas implica em uma maior taxa de transferência de calor para as partículas, o que leva a uma maior evaporação de água do produto, resultando em pós com umidade mais baixa. Quanto ao efeito da concentração de maltodextrina, de acordo com Abadio et al. (2004) e Quek et al. (2007), a

Tabela 2. Análise de variância (ANOVA) para cada uma das características físico-químicas das amostras produzidas por spray drying.

\begin{tabular}{ccccccccc} 
& SQ Efeitos & GL Efeitos & MQ Efeitos & SQ Erro & GL Erro & MQ Erro & F & P \\
U & 11,19 & 5 & 2,24 & 0,04 & 12 & 0,003 & 746,0 & $<0,05$ \\
Hi & 282,48 & 5 & 56,49 & 1,53 & 12 & 0,127 & 444,8 & $<0,05$ \\
Aw & 0,0015 & 5 & 0,0003 & 0,0001 & 12 & 0,000008 & 37,5 & $<0,05$ \\
TA & 235221,70 & 5 & 47044,35 & 221,91 & 12 & 18,493 & 2542,9 & $<0,05$ \\
D & 3335,86 & 5 & 667,17 & 63,11 & 50 & 1,262 & 528,5 & $<0,05$ \\
L $^{*}$ & 248,35 & 5 & 49,67 & 82,60 & 48 & 1,721 & 28,9 & $<0,05$ \\
a $^{*}$ & 244,06 & 5 & 48,81 & 93,72 & 48 & 1,953 & 25,0 & $<0,05$ \\
b* $^{*}$ & 34,61 & 5 & 6,92 & 1,69 & 48 & 0,035 & 197,7 & $<0,05$ \\
C* $^{*}$ & 260,77 & 5 & 52,15 & 91,20 & 48 & 1,899 & 27,5 & $<0,05$ \\
$H^{*}$ & 217,41 & 5 & 43,48 & 2,99 & 48 & 0,062 & 701,3 & $<0,05$ \\
\hline
\end{tabular}

$\mathrm{U}=$ umidade (\%); Hi = higroscopicidade ( $\left.\mathrm{g} .100 \mathrm{~g}^{-1}\right) ; \mathrm{Aw}=$ atividade de água; TA = teor de antocianinas (mg.100 g $\mathrm{g}^{-1} \mathrm{~b} . \mathrm{s}$.); $\mathrm{D}=$ diâmetro médio $(\mu \mathrm{m}) ; L^{*}, a^{*}, b^{*}, C^{*}$ e $H^{*}=$ parâmetros de cor; $S Q=$ soma quadrática; $G L$ = graus de liberdade; $M Q=$ média quadrática. 
Secagem por atomização de polpa de amora-preta usando maltodextrina como agente carreador

FERRARI, C. C. et al.

Tabela 3. Temperatura do ar de saída $\left(\mathrm{T}_{\text {saída }}\right)$, umidade, higroscopicidade e atividade de água das amostras de suco de amora-preta em pó, produzidas sob diferentes temperaturas de secagem ( $\mathrm{T}_{\text {entrada }}$ ) e concentrações de maltodextrina (M).

\begin{tabular}{|c|c|c|c|c|c|}
\hline $\begin{array}{c}\mathrm{T}_{\text {entrada }} \\
\left({ }^{\circ} \mathrm{C}\right)\end{array}$ & $\begin{array}{c}M \\
(\%)\end{array}$ & $\begin{array}{l}T_{\text {saida }} \\
\left({ }^{\circ} \mathrm{C}\right)\end{array}$ & $\begin{array}{l}\text { Umidade } \\
(\%)\end{array}$ & $\begin{array}{c}\text { Higroscopicidade } \\
\left(\mathrm{g} .100 \mathrm{~g} \mathrm{~g}^{-1}\right)\end{array}$ & $\begin{array}{l}\text { Atividade } \\
\text { de água }\end{array}$ \\
\hline 160 & 5 & 105 & $3,05 \pm 0,08^{a}$ & $28,73 \pm 0,30^{a}$ & $0,280 \pm 0,003^{a}$ \\
\hline 160 & 15 & 108 & $1,51 \pm 0,05^{b}$ & $22,32 \pm 0,41^{b}$ & $0,283 \pm 0,003^{a}$ \\
\hline 160 & 25 & 109 & $1,08 \pm 0,05^{\mathrm{cd}}$ & $18,77 \pm 0,28^{c}$ & $0,278 \pm 0,003^{a}$ \\
\hline 180 & 5 & 112 & $1,23 \pm 0,05^{d}$ & $29,51 \pm 0,16^{d}$ & $0,260 \pm 0,002^{b}$ \\
\hline 180 & 15 & 115 & $1,02 \pm 0,05^{c}$ & $23,01 \pm 0,28^{e}$ & $0,261 \pm 0,002^{b}$ \\
\hline 180 & 25 & 117 & $0,55 \pm 0,06^{e}$ & $20,78 \pm 0,57^{f}$ & $0,265 \pm 0,003^{b}$ \\
\hline
\end{tabular}

Letras diferentes na mesma coluna indicam diferença significativa entre os tratamentos para $p \leq 0,05$

Tabela 4. Teor de antocianinas, retenção de antocianinas e diâmetro médio das amostras de suco de amora-preta em pó, produzidas sob diferentes temperaturas de secagem $\left(\mathrm{T}_{\text {entrada }}\right)$ e concentrações de maltodextrina $(\mathrm{M})$.

\begin{tabular}{|c|c|c|c|c|}
\hline $\begin{array}{l}\mathrm{T}_{\text {entrada }} \\
\left({ }^{\circ} \mathrm{C}\right)\end{array}$ & $\begin{array}{c}M \\
(\%)\end{array}$ & $\begin{array}{l}\text { Teor antocianinas } \\
\text { (mg.100 } \mathrm{g}^{-1} \text { b.s.) }\end{array}$ & $\begin{array}{c}\text { Retenção } \\
\text { antocianinas (\%) }\end{array}$ & $\begin{array}{c}\text { Diâmetro médio } \\
\text { D }[4,3](\mu \mathrm{m})\end{array}$ \\
\hline 160 & 5 & $433,32 \pm 9,03^{a}$ & 80,56 & $34,18 \pm 1,98^{a}$ \\
\hline 160 & 15 & $208,52 \pm 1,01^{b}$ & 71,45 & $13,33 \pm 0,18^{b}$ \\
\hline 160 & 25 & $136,50 \pm 0,05^{c}$ & 76,80 & $14,85 \pm 0,59 c$ \\
\hline 180 & 5 & $360,08 \pm 1,73^{d}$ & 74,01 & $21,62 \pm 1,55^{d}$ \\
\hline 180 & 15 & $188,12 \pm 1,39^{e}$ & 69,79 & $14,10 \pm 0,17^{b c}$ \\
\hline 180 & 25 & $129,33 \pm 0,28^{f}$ & 69,09 & $26,42 \pm 0,81^{e}$ \\
\hline
\end{tabular}

Letras diferentes na mesma coluna indicam diferença significativa entre os tratamentos para $p \leq 0,05$

Tabela 5. Parâmetros de cor das amostras de suco de amora-preta em pó, produzidas sob diferentes temperaturas de secagem $\left(T_{\text {entrada }}\right)$ e concentrações de maltodextrina $(M)$.

\begin{tabular}{ccccccc}
$\mathbf{T}_{\text {entrada }}\left({ }^{\circ} \mathbf{C}\right)$ & $\mathbf{M}(\%)$ & $\mathbf{L}^{*}$ & $\mathbf{a}^{*}$ & $\mathbf{b}^{*}$ & $\mathbf{C}^{*}$ & $\mathbf{H}^{*}$ \\
160 & 5 & $34,74 \pm 0,43^{\mathrm{a}}$ & $19,79 \pm 1,03^{\mathrm{a}}$ & $3,81 \pm 0,25^{\mathrm{a}}$ & $20,16 \pm 1,05^{\mathrm{a}}$ & $10,91 \pm 0,39^{\mathrm{a}}$ \\
160 & 15 & $39,35 \pm 1,61^{\mathrm{b}}$ & $18,20 \pm 1,73^{\mathrm{a}}$ & $2,28 \pm 0,33^{\mathrm{b}}$ & $18,32 \pm 1,74^{\mathrm{a}}$ & $6,72 \pm 0,15^{\mathrm{b}}$ \\
160 & 25 & $39,93 \pm 2,48^{\mathrm{b}}$ & $17,43 \pm 2,44^{\mathrm{a}}$ & $1,71 \pm 0,18^{\mathrm{c}}$ & $17,51 \pm 2,45^{\mathrm{a}}$ & $5,63 \pm 0,25^{\mathrm{c}}$ \\
180 & 5 & $35,18 \pm 0,22^{\mathrm{a}}$ & $19,18 \pm 0,37^{\mathrm{a}}$ & $2,63 \pm 0,07^{\mathrm{d}}$ & $19,29 \pm 0,35^{\mathrm{a}}$ & $9,77 \pm 0,20^{\mathrm{d}}$ \\
180 & 15 & $39,56 \pm 0,73^{\mathrm{b}}$ & $19,68 \pm 0,85^{\mathrm{a}}$ & $2,66 \pm 0,11^{\mathrm{d}}$ & $19,86 \pm 0,86^{\mathrm{a}}$ & $7,70 \pm 0,18^{\mathrm{e}}$ \\
180 & 25 & $36,48 \pm 0,50^{\mathrm{c}}$ & $13,60 \pm 0,40^{\mathrm{b}}$ & $1,29 \pm 0,06^{\mathrm{e}}$ & $13,65 \pm 0.44^{\mathrm{b}}$ & $5,62 \pm 0,22^{\mathrm{c}}$ \\
\hline
\end{tabular}

Letras diferentes na mesma coluna indicam diferença significativa entre os tratamentos para $p \leq 0,05$

adição de maltodextrina antes da secagem em spray dryer aumenta o conteúdo de sólidos totais da mistura a ser alimentada no spray drying, reduzindo a quantidade de água a ser evaporada, o que acarreta a diminuição da umidade dos pós produzidos.

Em relação à higroscopicidade, maiores concentrações de maltodextrina resultaram em pós menos higroscópicos, o que está associado ao fato de a maltodextrina ser um material com baixa higroscopicidade, reduzindo a capacidade de adsorção de água dos produtos desidratados em spray dryer. Valores de higroscopicidade significativamente maiores $(p \leq 0,05)$ foram observados nas amostras produzidas a $180{ }^{\circ} \mathrm{C}$. Segundo Tonon et al. (2008), temperaturas de secagem mais altas resultam em pós com umidades mais baixas e maior facilidade em adsorver água, ou seja, mais higroscópicos, o que está relacionado ao maior gradiente de concentração de água existente entre o produto e o ambiente. Rodriguez-Hernandez et al. (2005), em um estudo com secagem em spray dryer de figo-da-índia, utilizando concentrações de maltodextrina de 18-23\% e temperatura de secagem de $205-225^{\circ} \mathrm{C}$, obtiveram pós mais higroscópicos com as menores concentrações de maltodextrina e maiores temperaturas de secagem; tais ocorrências também foram verificadas no presente trabalho.

\subsubsection{Retenção de antocianinas e distribuição do tamanho de partículas}

De acordo com a Tabela 4, o aumento da temperatura do ar de secagem e da concentração de maltodextrina levou à diminuição estatisticamente significativa da concentração de antocianinas presente no suco de amora-preta em pó. As amostras produzidas com temperatura de secagem de $180^{\circ} \mathrm{C}$ e $25 \%$ de maltodextrina foram as que apresentaram menor teor de antocianinas 
após o processo (em torno de $130 \mathrm{mg} .100 \mathrm{~g}^{-1}$ b.s.). Cai e Corke (2000), analisando a retenção de betacianinas extraídas de amaranto, reportaram que temperaturas altas proporcionam uma maior perda dos pigmentos, apesar da maior velocidade de secagem e rendimento. Segundo Quek et al. (2007), pós secos em temperaturas mais baixas possuem uma tendência à aglomeração, em razão do maior teor de umidade, especialmente em amostras com elevado teor de açúcar. A aglomeração faz com que as partículas apresentem menor superfície exposta, diminuindo a exposição dos pós ao oxigênio e protegendo os pigmentos contra a degradação. Os autores verificaram uma redução de 30\% no teor de licopeno em suco de melancia em pó obtido por spray drying, ao se aumentar a temperatura de secagem de 145 para $175^{\circ} \mathrm{C}$.

Em relação ao efeito da maltodextrina, maiores concentrações de agente carreador na mistura a ser alimentada no spray dryer provocam um efeito de diluição dos pigmentos presentes na amostra, havendo perda de nutrientes e da cor do produto, o que diminui a qualidade dos pós produzidos. Na medida em que o teor de sólidos totais da polpa de amora-preta é em torno de $8 \%$ (Tabela 1), ao se acrescentar $25 \%$ de maltodextrina na mistura a ser atomizada, a quantidade de agente carreador presente no pó é de aproximadamente $75 \%$. Por outro lado, trabalhando-se com $5 \%$ de maltodextrina, cerca de $40 \%$ dos sólidos totais do pó são provenientes do agente carreador. Esses cálculos explicam as grandes diferenças observadas no teor de antocianinas dos pós de amora-preta (Tabela 4), ilustrando o efeito de diluição das antocianinas ocasionado pelo aumento da concentração de agente carreador. Em um trabalho semelhante, Kha et al. (2010) verificaram que o aumento da concentração de maltodextrina de 10 para $30 \%$ proporcionou uma diminuição do conteúdo total de carotenoides de $195 \mathrm{mg} .100 \mathrm{~g}^{-1}$ para $61 \mathrm{mg} .100 \mathrm{~g}^{-1} \mathrm{em}$ gac fruit produzida por spray drying.

A retenção de antocianinas foi cerca de $80 \%$ apenas para as amostras produzidas com $5 \%$ de maltodextrina e temperatura de secagem de $160{ }^{\circ} \mathrm{C}$, mostrando o efeito sinergístico dessas duas variáveis na manutenção do teor de antocianinas da amora-preta. Por outro lado, nos ensaios realizados com 15 e $25 \%$ de maltodextrina, e temperatura de $180^{\circ} \mathrm{C}$, foi constatada uma retenção em torno de apenas $69 \%$ dos pigmentos da amora-preta após a secagem, uma vez que as antocianinas são pigmentos termossensíveis.

Em relação ao tamanho de partícula, os menores diâmetros médios foram observados para as amostras produzidas com $15 \%$ de maltodextrina e temperatura de $180^{\circ} \mathrm{C}$, não diferindo estatisticamente - $p \leq 0,05$ - dos valores dos pós obtidos usando temperatura de secagem de $160{ }^{\circ} \mathrm{C}$ e concentrações de maltodextrina de 15 ou $25 \%$
(Tabela 4). O aumento da temperatura de secagem resultou em maiores diâmetros médios para as partículas produzidas com concentrações de maltodextrina de 25\% (Tabela 4). Tonon et al. (2008) e Nijdam e Langrish (2006), trabalhando: com açaí e leite em pó, respectivamente, produzidos por spray drying, também verificaram a formação de partículas maiores com o aumento da temperatura do processo. Segundo os autores, ao se utilizarem maiores temperaturas no processo de secagem por atomização, o tempo de secagem é menor, promovendo a formação mais rápida de uma estrutura, o que evita o encolhimento das partículas durante a secagem. Em temperaturas mais baixas, a partícula encolhe e, consequentemente, o diâmetro diminui, o que justifica os resultados obtidos no presente trabalho. No entanto, verificou-se um comportamento distinto no ensaio realizado com $5 \%$ de maltodextrina e temperatura de $160{ }^{\circ} \mathrm{C}$, visto que as partículas apresentaram um diâmetro médio significativamente maior (em torno de $34 \mu \mathrm{m})$. Tal fato pode ser atribuído à maior umidade observada nessa condição, em comparação aos outros ensaios (Tabela 3), já que pós com umidades mais altas possuem uma maior tendência em aglomerar, o que pode resultar em partículas maiores.

Avaliando-se a influência do agente carreador, partículas com diâmetros médios significativamente maiores foram obtidas com o aumento da concentração de maltodextrina de 15 para $25 \%$, fato que pode ser relacionado ao aumento da viscosidade da mistura alimentada no spray dryer. Tal aumento foi mais acentuado nos ensaios realizados a $180{ }^{\circ} \mathrm{C}$, provavelmente em função do efeito combinado do uso de alta temperatura de secagem e de maior concentração de maltodextrina. De acordo com Goula e Adamopoulos (2004), quando a viscosidade da solução aumenta, maiores são as gotículas formadas na atomização, o que resulta na formação de partículas maiores.

A Figura 1 apresenta a distribuição do tamanho de partículas produzidas por spray drying. As partículas apresentaram tamanhos diversos, com diâmetros variando de 0,1 a $410 \mu \mathrm{m}$, aproximadamente. Observa-se uma distribuição bimodal, ou seja, dois picos distintos no gráfico, indicando a presença de dois tamanhos predominantes, sendo que o primeiro pico representa as partículas de menor diâmetro (em torno de $1 \mu \mathrm{m}$ ), enquanto que o pico maior indica as partículas com maior diâmetro. Todas as curvas apresentaram comportamentos semelhantes, com frações volumétricas do tamanho de partículas entre 8 e $11 \%$. Entretanto, no ensaio realizado com $25 \%$ de maltodextrina e temperatura de secagem de $180{ }^{\circ} \mathrm{C}$, o pico principal apresentou um menor volume (menor que 6\%) e a curva se mostrou mais larga em relação às outras, sugerindo uma maior uniformidade do tamanho das partículas nessa condição. 


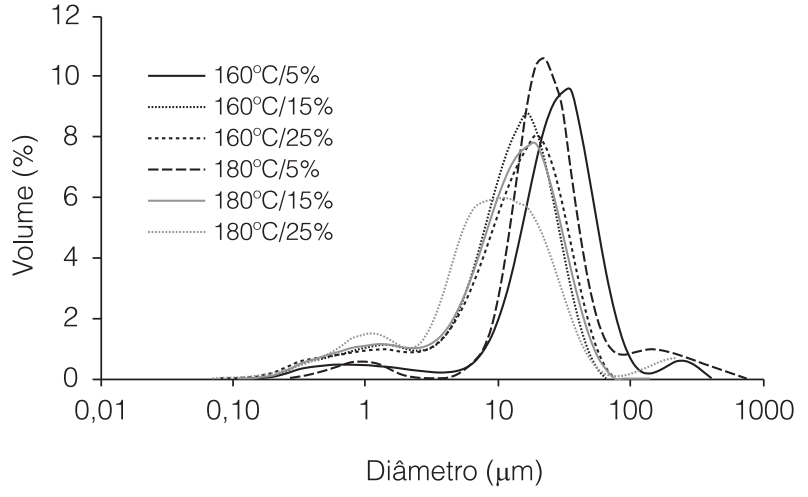

Figura 1. Distribuição do tamanho de partículas das amostras de suco de amora-preta em pó, produzidas utilizando diferentes temperaturas de secagem $\left(160^{\circ} \mathrm{C}\right.$ ou $\left.180^{\circ} \mathrm{C}\right)$ e concentrações de maltodextrina (5, 15 ou $25 \%$ ).

\subsubsection{Microestrutura}

A Figura 2 apresenta as imagens microscópicas das partículas de suco de amora-preta em pó obtido por spray drying. De maneira geral, as partículas mostraram formato esférico, com diversos tamanhos. Nos tratamentos realizados com temperatura de secagem de $160^{\circ} \mathrm{C}$, observou-se que todas as amostras apresentaram superfície lisa, com exceção do ensaio realizado com $15 \%$ de maltodextrina. Nesse caso, as partículas se mostraram mais murchas e enrugadas (círculos pretos - Figura $2 \mathrm{~b}$ ), havendo um maior espaçamento entre elas. Essas diferenças na morfologia das partículas podem ser associadas aos maiores valores de retenção de antocianinas verificados para os tratamentos conduzidos com 5 e $25 \%$ de maltodextrina (em torno de 80 e $76 \%$, respectivamente), se comparados ao ensaio com $15 \%$ de maltodextrina (aproximadamente $71 \%$ ), de acordo com a Tabela 4. Uma maior aderência (aglomeração) das partículas diminui a exposição dos pós ao oxigênio, reduzindo a oxidação dos pigmentos, conforme já explicado anteriormente no item 3.2.1.

As partículas produzidas com temperatura de secagem de $180{ }^{\circ} \mathrm{C}$ apresentaram superfície lisa e uma maior uniformidade em relação às amostras secas à temperatura de $160{ }^{\circ} \mathrm{C}$. No entanto, apesar de a estrutura ser mais homogênea, os pós produzidos com temperaturas de secagem mais altas $\left(180^{\circ} \mathrm{C}\right)$ apresentaram menor retenção de antocianinas (Tabela 4), indicando que o efeito da temperatura de secagem na retenção de antocianinas foi mais intenso do que o efeito dessa mesma variável na morfologia das partículas. De acordo com Nijdam e Langrish (2006), em temperaturas mais altas, a água evapora mais rapidamente e a película formada se torna seca e rígida, impedindo que as partículas encolham e, assim, como resultado, há uma estrutura mais uniforme. Os autores observaram a formação de esferas mais lisas e rígidas em leite em pó obtido por spray dryer, à temperatura de $200^{\circ} \mathrm{C}$, enquanto que o leite produzido a $120{ }^{\circ} \mathrm{C}$ apresentou partículas menores e com aparência mais rugosa.

\subsubsection{Cor}

Os resultados das análises de cor das amostras submetidas aos diferentes ensaios de secagem por spray dryer são apresentados na Tabela 5.

Maiores concentrações de maltodextrina resultaram em um aumento significativo nos valores de $L^{*}$ das amostras secas a $160{ }^{\circ} \mathrm{C}$, enquanto que, nos ensaios realizados a $180{ }^{\circ} \mathrm{C}$, observou-se uma diminuição estatisticamente dos resultados desse parâmetro ao se aumentar a concentração de maltodextrina de 15 para $25 \%$. Kha et al. (2010) também observaram um aumento do parâmetro $L^{*}$ com o aumento da concentração de maltodextrina em gac fruit seca em spray dryer, uma vez que a maltodextrina possui coloração branca e, desse modo, dilui os pigmentos presentes na fruta, alterando a sua coloração. A temperatura mostrou efeito significativo na redução da luminosidade somente nos ensaios realizados com maior concentração de maltodextrina (25\%). Tal fato pode ser relacionado à maior degradação de antocianinas, em função do menor conteúdo e da menor retenção de pigmentos verificados nessa condição (Tabela 4). Ahmed et al. (2010) atribuíram as diferenças na cor de batata-doce-roxa em pó obtida por spray drying à presença de antocianinas poliméricas, as quais possuem tonalidade mais marrom, resultando em pós mais escuros (menor $L^{*}$ ), decorrentes de reações de degradação.

O parâmetro $a^{*}$ apresentou redução significativa apenas nas amostras secas com $25 \%$ de maltodextrina à temperatura de $180^{\circ} \mathrm{C}$, denotando uma diminuição da tonalidade vermelha. No caso do parâmetro $b^{*}$, menores valores foram observados com o aumento da temperatura e da concentração de maltodextrina, o que mostra uma intensificação da tonalidade azul dos pós. O croma C* seguiu a mesma tendência dos valores do parâmetro $a^{*}$, indicando que o parâmetro $a^{*}$ foi mais expressivo na determinação do croma do suco de amora-preta em pó. Uma diminuição significativa dos valores de $C^{*}$ foi verificada apenas para as amostras secas a $180{ }^{\circ} \mathrm{C}$ com $25 \%$ de maltodextrina, resultando em um pó com coloração menos viva, o que pode ser atribuído à maior destruição dos pigmentos nessa condição. O ângulo de tom $\mathrm{H}^{\star}$ apresentou uma diminuição estatisticamente significativa com o aumento da concentração de maltodextrina, mostrando uma tonalidade mais roxa dos pós, por causa da redução da intensidade da cor vermelha (menores valores de $a^{*}$ ) e do aumento da intensidade da cor azul (menores valores de $b^{\star}$ ). Tal ocorrência também foi verificada por Ahmed et al. (2010) e Ersus e Yurdagel (2007) em batata-doce-roxa e black carrot, respectivamente, secas em spray dryer. 

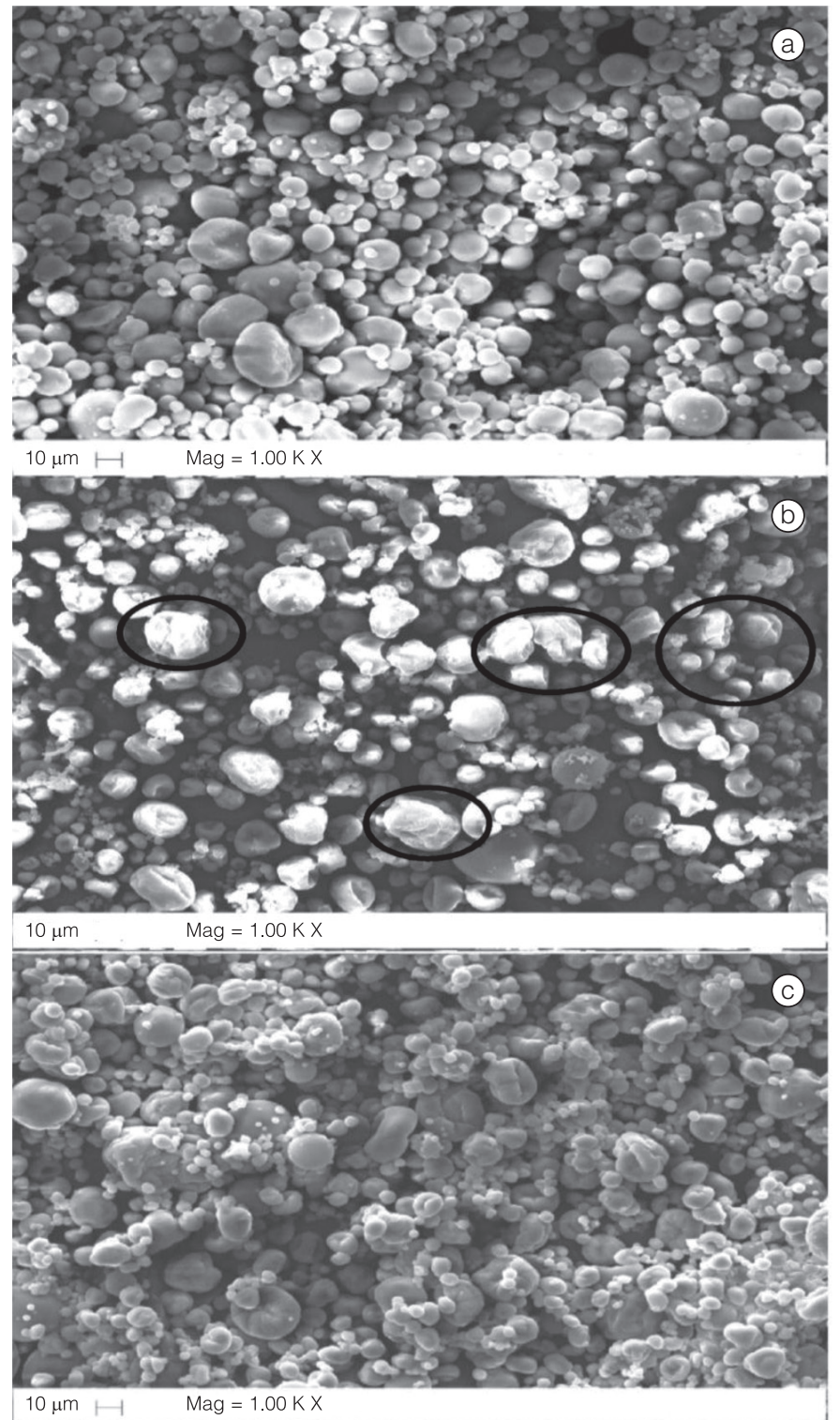
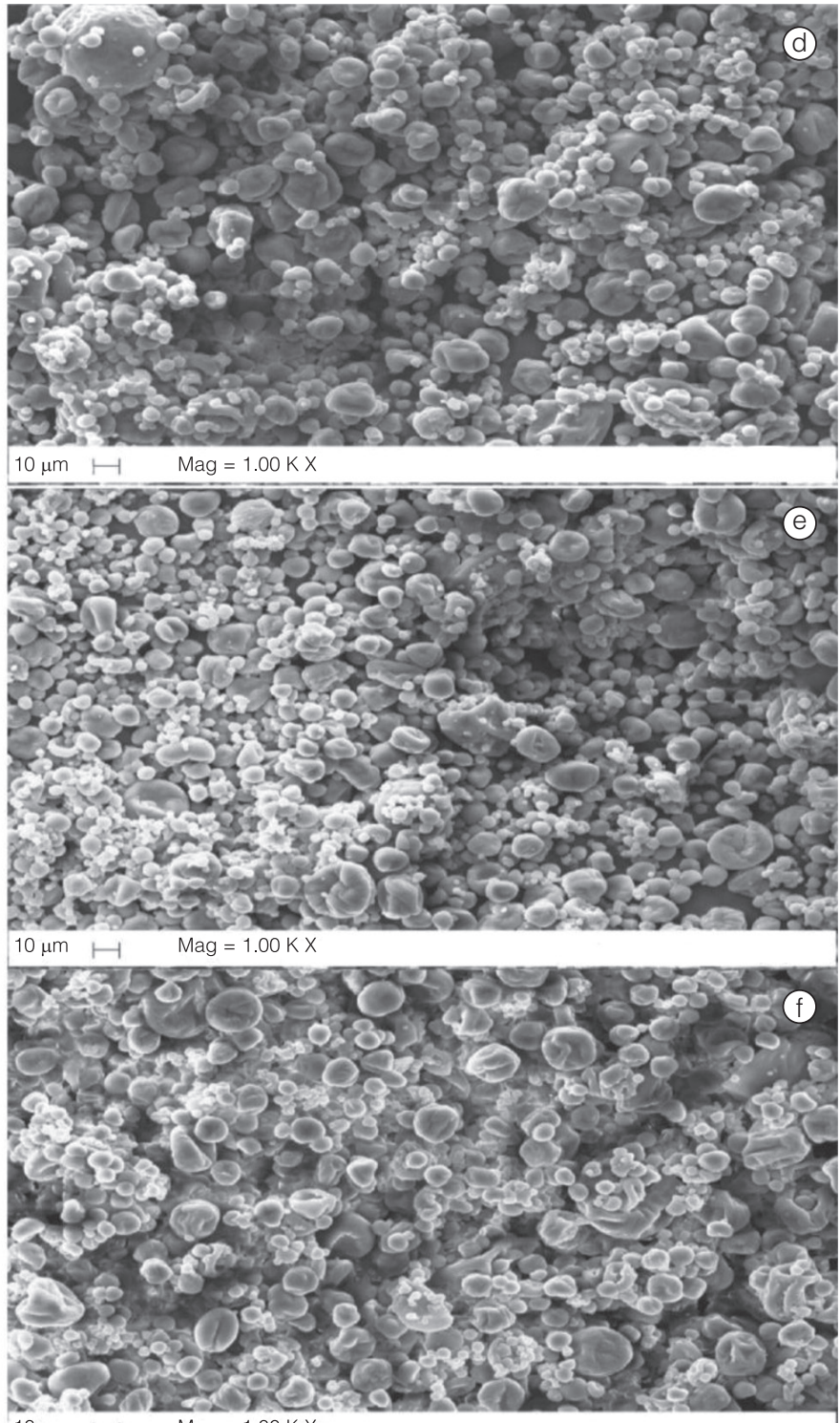

$0 \mu \mathrm{m} \mapsto \mathrm{Mag}=1.00 \mathrm{KX}$

Figura 2. Imagens microscópicas das amostras de suco de amora-preta em pó, produzidas sob diferentes temperaturas de secagem: (a), (b) e (c): $160{ }^{\circ} \mathrm{C}$; (d), (e) e (f): $180{ }^{\circ} \mathrm{C}$ e diferentes concentrações de maltodextrina: (a) e (d): 5\%; (b) e (e): 15\%; (c) e (f): $25 \%$, com um aumento de 1000 vezes. Barra $=10 \mu \mathrm{m}$.

\section{Conclusões}

O aumento da temperatura do ar de secagem resultou em pós menos úmidos e com menor atividade de água, porém mais higroscópicos e com menor conteúdo de antocianinas. Além disso, essa variável teve uma influência significativa na diminuição dos valores dos parâmetros de cor $L^{*}$ e $C^{*}$ somente nos ensaios realizados com $25 \%$ de maltodextrina. De maneira geral, as partículas produzidas à temperatura de $180{ }^{\circ} \mathrm{C}$ apresentaram tamanho maior, superfície lisa e uma maior uniformidade. O aumento da concentração de maltodextrina resultou na formação de partículas com menor teor de umidade e higroscopicidade. Esses pós também apresentaram menor teor de antocianinas, o que acarretou o aumento da luminosidade e a redução do croma e do ângulo de tom das amostras. O ensaio realizado à temperatura de $160{ }^{\circ} \mathrm{C}$ com $5 \%$ de maltodextrina foi o mais efetivo na manutenção do teor de antocianinas do suco de amorapreta em pó, mostrando uma retenção destes pigmentos em torno de $80 \%$.

\section{Agradecimentos}

Os autores agradecem à Fundação de Amparo à Pesquisa do Estado de São Paulo (FAPESP - processo $n^{\circ}$ 2010/02561-1) pelo apoio financeiro.

\section{Referências}

ABADIO, F. D. B.; DOMINGUES, A. M.; BORGES, S. V.; OLIVEIRA, V. M. Physical properties of powdered pineapple 
Secagem por atomização de polpa de amora-preta usando maltodextrina como agente carreador

FERRARI, C. C. et al.

(Ananás comosus) juice - effect of malt dextrin concentration and atomization speed. Journal of Food Engineering, Oxford, v. 64, n. 3, p. 285-287, 2004. http://dx.doi.org/10.1016/j. jfoodeng.2003.10.010

ACOSTA-MONTOYA, O.; VAILLANT, F.; COZZANO, S.; MERTZ, C.; PÉREZ, A. M. Phenolic content and antioxidant capacity of tropical highland blackberry (Rubus adenotrichus Schltdl.) during three edible maturity stages. Food Chemistry, Barking, v. 119, n. 4, p. 1497-1501, 2010. http://dx.doi.org/10.1016/j. foodchem.2009.09.032

AHMED, M.; AKTER, M. S.; LEE, J. C.; EUN, J. B. Encapsulation by spray drying of bioactive components, physicochemical and morphological properties from purple sweet potato. LWT - Food Science and Technology, Zurich, v. 43, n. 9, p. 1307-1312, 2010. http://dx.doi.org/10.1016/j.Iwt.2010.05.014

BHANDARI, B. R.; DATTA, N.; HOWES, T. Problems associated with spray drying of sugar-rich foods. Drying Technology, Philadelphia, v. 15, n. 2, p. 671-684, 1997. http://dx.doi. org/10.1080/07373939708917253

CAI, Y. Z.; CORKE, H. Production and properties of spraydried Amaranthus betacyanin pigments. Journal of Food Science, Chicago, v. 65, n. 6, p. 1248-1252, 2000. http://dx.doi. org/10.1111/j.1365-2621.2000.tb10273.x

CALVO, C.; DURÁN, L. Propiedades Físicas II - Ópticas y Color. In: AGUILERA, J. M. (Ed.). Temas en Tecnología de Alimentos. Cidade do México: Instituto Politécnico Nacional, 1997. v. 1, cap. 7, p. 261-288.

ERSUS, S.; YURDAGEL, U. Microencapsulation of anthocyanin pigments of black carrot (Daucuscarota L.) by spray dryer. Journal of Food Engineering, Oxford, v. 80, n. 3, p. 805-812, 2007. http://dx.doi.org/10.1016/j.jfoodeng.2006.07.009

FERREIRA, D. S. Compostos Bioativos em Amora-Preta e Encapsulação do seu Extrato Antociânico por Gelificação Térmica com Curdlana. 2008. 88 f. Dissertação (Mestrado em Ciência de Alimentos)-Faculdade de Engenharia de Alimentos, Universidade Estadual de Campinas, Campinas, 2008.

GOULA, A. M.; ADAMOPOULOS, K. G. Spray drying of tomato pulp: effect of feed concentration Drying Technology, Philadelphia, v. 22, n. 10, p. 2309-2330, 2004. http://dx.doi. org/10.1081/DRT-200040007

HORWITZ, W. (Ed.). Official Methods of Analysis of AOAC International. 18th ed. Maryland: AOAC Press, 2006.
KHA, T. C.; NGUYEN, M. H.; ROACH, P. D. Effects of spray drying conditions on the physicochemical and antioxidant properties of the Gac (Momordica cochinchinensis) fruit aril powder. Journal of Food Engineering, Oxford, v. 98, n. 3, p. 385-392, 2010. http://dx.doi.org/10.1016/j.jfoodeng.2010.01.016

KIRALP, S.; TOPPARE, L. Polyphenol content in selected turkish wines, an alternative method of detection of phenolics. Process Biochemistry, Oxford, v. 41, n. 1, p. 236-239, 2006. http://dx.doi. org/10.1016/j.procbio.2005.06.011

MOREIRA, G. E. G.; COSTA, M. G. M.; SOUZA, A. C. R.; BRITO, E. S.; MEDEIROS, M. F. D.; AZEREDO, H. M. C. Physical properties of spray dried acerola pomace extract as affected by temperature and drying aids. LWT - Food Science and Technology, Zurich, v. 42, n. 2, p. 641-645, 2009. http://dx.doi. org/10.1016/j.Iwt.2008.07.008

NIJDAM, J. J.; LANGRISH, T. A. J. The effect of surface composition on the functional properties of milk powders. Journal of Food Engineering, Oxford, v. 77, n. 4, p. 919-925, 2006. http://dx.doi.org/10.1016/j.jfoodeng.2005.08.020

QUEK, S. Y.; CHOK, N. K.; SWEDLUND, P. The physicochemical properties of spray-dried watermelon powders. Chemical Engineering and Processing. Amsterdam, v. 46, n. 5, p. 386-392, 2007. http://dx.doi.org/10.1016/j.cep.2006.06.020

RÉ, M. I. Microencapsulation by spray drying. Drying Technology, Philadelphia, v. 16, n. 6, p. 1195-1236, 1998. http:// dx.doi.org/10.1080/07373939808917460

RODRIGUEZ-HERNANDEZ, G. R.; GONZÁLEZ-GARCÍA, R.; GRAJALES-LAGUNES, A.; RUIZ-CABRERA, M. A. Spraydrying of cactus pear juice (Opuntia streptacantha): Effect on the physicochemical properties of powder and reconstituted product. Drying Technology, Philadelphia, v. 23, n. 4, p. 955-973, 2005. http://dx.doi.org/10.1080/DRT-200054251

TONON, R. V.; BRABET, C.; HUBINGER, M. D. Influence of process conditions on the physicochemical properties of açaí (Euterpe oleraceae Mart.) powder produced by srapy drying. Journal of Food Engineering, Oxford, v. 88, n. 3, p. 411-418, 2008. http://dx.doi.org/10.1016/j.jfoodeng.2008.02.029

VIZZOTTO, M. Amora-Preta: uma Fruta Antioxidante. Embrapa, 2008. Disponível em: <http://www.cpact.embrapa.br/ imprensa/artigos/artigos_2008.php/>. Acesso em: 28 jan. 2010. 